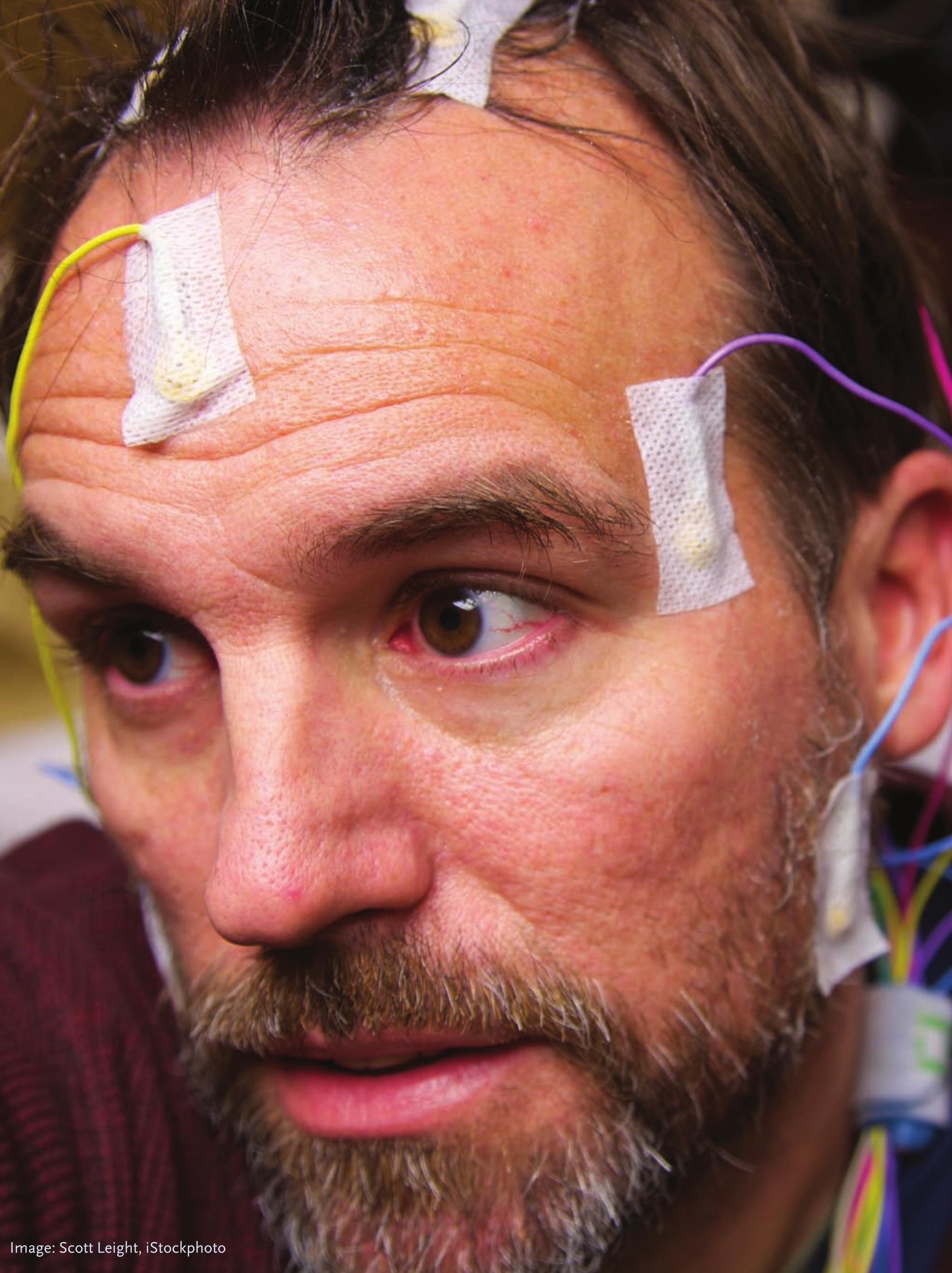




\begin{tabular}{|c|c|c|c|}
\hline & $\square$ & $\equiv$ & (2) \\
\hline B.D. Kent ${ }^{1,2}$ & 'Pulmonary and Sleep & Respiratory Sleep Disorders & walter.mcnicholas@ucd.ie \\
\hline W.T. McNicholas ${ }^{1,2}$ & $\begin{array}{l}\text { Disorders Unit, St Vincent's } \\
\text { University Hospital } \\
{ }^{2} \text { School of Medicine and } \\
\text { Medical Science, University } \\
\text { College Dublin, Dublin, } \\
\text { Ireland }\end{array}$ & $\begin{array}{l}\text { Unit, St Vincent's University } \\
\text { Hospital, Elm Park, Dublin } \\
\text { 4, Ireland }\end{array}$ & \\
\hline
\end{tabular}

\section{Ambulatory investigation of sleep-disordered breathing}

\section{Educational aims}

○ To outline the importance of improved diagnosis of sleep-disordered breathing and the need for ambulatory monitoring.

○ To discuss the currently available options for home monitoring and the recommendations for their use, as well as novel modalities.

$\odot$ To outline the issues surrounding patient selection and the need for further study.

Obstructive sleep apnoea syndrome (OSAS) is under-diagnosed, and attended inpatient sleep studies are resource intensive and inconvenient for the patient; therefore, there is increasing interest in the ambulatory diagnosis of OSAS. Full unattended polysomnography does not confer any advantage over more limited measurements. Ambulatory diagnosis of OSAS with cardiorespiratory polygraphy is suitable for use in patients with a high pre-test probability. Ambulatory monitoring by oximetry or ECG are not recommended by current guidelines, but a number of novel diagnostic modalities show some promise. Further studies are needed to address issues of cost-effectiveness, patient selection and device performance.

Obstructive sleep apnoea syndrome (OSAS) is a highly prevalent disorder, affecting at least $4 \%$ of middle-aged males and $2 \%$ of middleaged females [1]. Undiagnosed and untreated, OSAS leads to a significant reduction in quality of life, increased risk of road traffic accidents and increased incidence of depressive illness. However, in public health terms, the principal adverse consequence of OSAS is cardiovascular disease, with strong evidence suggesting that a failure to successfully identify and treat OSAS leads to markedly increased cardiovascular morbidity and mortality $[2,3]$. Furthermore, OSAS is independently associated with metabolic dysfunction and diabetes mellitus [4]. Unfortunately, OSAS is significantly underdiagnosed, limiting the scope for the introduction of potentially beneficial treatment. While factors limiting more widespread diagnosis of the disorder include a lack of public awareness,
Statement of interest None declared.

HERMES syllabus link: B.19.1, D.1.12 
and an acceptance by patients of their symptoms as normal variants, a key obstacle remains access to diagnostic facilities [5]. In-patient, attended polysomnography (PSG) remains the gold standard for diagnosis of sleep-disordered breathing, but is resource intensive and inconvenient for the patient. Unsurprisingly then, the development and validation of new diagnostic modalities permitting outpatient testing was identified as a research priority in the recent report from an expert taskforce established by the American Thoracic Society, the American Academy of Sleep Medicine (AASM), the American College of Chest Physicians and the European Respiratory Society [6].

This review will discuss the need for ambulatory diagnostic modalities in OSAS, the different technologies available and the evidence base evaluating the use of these devices before assessing where emerging technologies fit into the current diagnostic armamentarium.

\section{Why ambulatory investigation of OSAS?}

At least $4 \%$ of the adult male population of developed countries has clinically significant sleep-disordered breathing [7]. Based on data from the Wisconsin Sleep Cohort, $>80 \%$ of these individuals remain undiagnosed [8]. Meanwhile, although the prevalence of OSAS is lower in females, $<10 \%$ of those with OSAS will have been successfully identified [8]. Bearing in mind the rising tide of obesity engulfing the developed world, and the intimate relationship between obesity and sleep-disordered breathing, these prevalence figures probably represent conservative estimates. However, despite the marked under-recognition of the disorder, access to diagnostic sleep facilities is limited and characterised by unacceptably long waiting times. Indeed, were all individuals with moderate-severe OSAS to attend for assessment, $>600$ sleep studies per 100,000 population

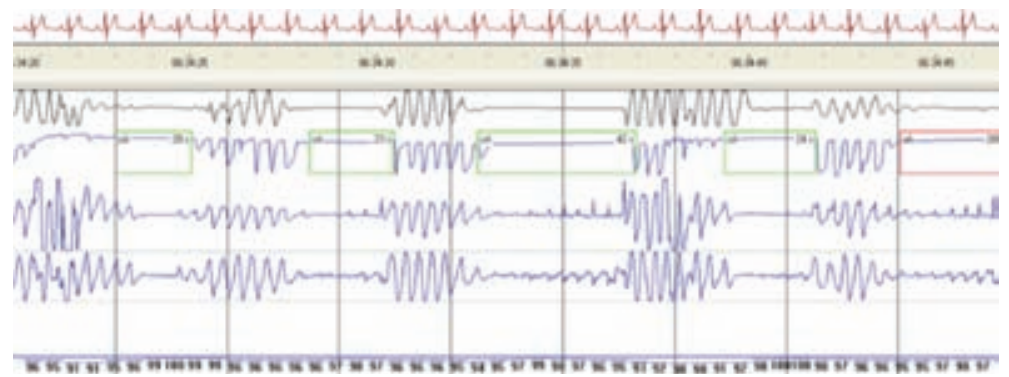

per annum would be required merely to deal with incident cases, vastly exceeding availability in all Western European countries and comfortably surpassing the combined diagnostic capacity of all North American sleep laboratories [5].

Furthermore, quite apart from being inconvenient for the patient, in-laboratory PSC with recording of physiological signals assessing sleep stage and cardiorespiratory parameters during sleep, is expensive, resource intensive and requires the supervision of a trained sleep technician.

This combination of a common disorder with limited diagnostic facilities has been a key driver of the burgeoning research interest in ambulatory diagnosis of OSAS. However, as we shall see, the current evidence base supporting the use of such techniques remains rather weak.

\section{What methods are available for the ambulatory diagnosis of OSAS?}

The first guidelines on the use of portable diagnostic equipment were published by the American Sleep Disorders Association (now the AASM) in 1994 [9]. These stratified diagnostic modalities for OSAS into four levels of complexity (table 1). This classification ranges from full, in-patient, attended PSG (type 1), with a minimum of seven signals, to unattended one- or two-signal recording devices, generally measuring oxygen saturation or airflow (type 4). While PSG remains the recommended gold standard, a substantial number of European sleep laboratories utilise four- to six-channel cardiorespiratory polygraphy (equivalent to type 3) as their primary in-patient diagnostic test. As will be discussed in detail below, the vast majority of ambulatory testing is performed using type 3 and 4 devices.

\section{Ambulatory diagnosis of OSAS with home polysomnography}

The use of unattended home PSC would seem an obvious solution to long waiting times for in-patient assessment. Indeed, using the same diagnostic methodology as the Sleep Heart Health Study, IBER et al. [10] found excellent 
Table 1 American Academy of Sleep Medicine classification of diagnostic modalities

\begin{tabular}{llll}
\hline $\begin{array}{l}\text { Test } \\
\text { level }\end{array}$ & Description & Personnel & Signals required \\
\hline Type 1 & $\begin{array}{l}\text { In-patient PSG performed } \\
\text { in a sleep laboratory }\end{array}$ & Attended & $\begin{array}{l}\text { EEG, EOG, chin EMG, ECG, airflow, } \\
\text { respiratory effort and oxygen saturation }\end{array}$ \\
\hline Type 2 & Portable PSG & Unattended & Same as type 1 \\
\hline Type 3 & $\begin{array}{l}\text { Cardiorespiratory } \\
\text { polygraphy }\end{array}$ & $\begin{array}{l}\text { Attended and } \\
\text { unattended }\end{array}$ & $\begin{array}{l}\text { 4-6 signals, including ECG or heart rate, } \\
\text { and oxygen saturation }\end{array}$ \\
\hline Type 4 & $\begin{array}{l}\text { 1-2 signals } \\
\text { PSG: polysomnography; EEG: electroencephalography; EOG: electro-oculography; EMG: electromyography. }\end{array}$
\end{tabular}

agreement between home and laboratory PSC estimates of sleep-disordered breathing, while patients had better sleep time and efficiency in their own beds than in a sleep laboratory. However, other groups have found significant issues with the reliability of data obtained by home PSG recordings. For example, in a study of home and in-patient PSC conducted on 103 subjects in a single French sleep laboratory, $20 \%$ of home studies had to be excluded from analysis due to poor data quality [11]. Similarly, sleep staging and arousal data were deemed unreliable in a quarter of unattended PSC recordings from the Sleep Heart Health Study [12]. Therefore, unless actually attended by a trained sleep technician, there appears to be little added benefit from full home PSC over more limited measurement of cardiorespiratory parameters.

\section{Ambulatory diagnosis of OSAS with cardiorespiratory
polygraphy}

A substantial number of studies have explored the use of four- to six-channel (type 3) devices in a domiciliary setting. The majority of these systems combine pulse oximetry with measurement of airflow, respiratory effort and body position. Initial evidence-based, systematic reviews of this technology concluded that its use was associated with unacceptably high false-positive and false-negative results, and recommended against their use [13]. However, with improvements in technology have come improvements in diagnostic accuracy, and the most recent AASM clinical guidelines concluded that the use of unattended portable systems is feasible under certain circumstances [14]. In particular, home testing was deemed appropriate in subjects with a high pre-test probability of OSAS, and then only if the investigation of these patients was under the supervision of a dedicated sleep physician, with the diagnostic device applied by an experienced sleep technician. Conversely, the guidelines recommended that patients with a low pre-test probability of OSAS, those with significant comorbid medical illness or those with suspected non-OSAS sleep disorders should undergo formal in-patient PSC as their initial investigation. Similarly, a negative or technically inadequate home sleep study should prompt overnight admission to the sleep laboratory for further investigation.

\section{Ambulatory diagnosis of OSAS with type 4 devices}

\section{Oximetry}

The majority of type 4 systems utilise pulse oximetry, with or without concomitant measurement of ECG or oronasal airflow. Oximetry is an attractive, intuitive diagnostic option that has been found by some investigators to predict outcomes of treatment of OSAS at least as well as in-patient PSG [15]. However, oxyhaemoglobin desaturation may be absent in hypopnoeas and obstruction-induced arousals, and in non-obese subjects. Furthermore, 
the diagnostic accuracy of oximetry may be further compromised by the use of one of the less expensive commercially available oximeters [12]. A comprehensive review of the published literature found a wide variety in the reported sensitivity and specificity of oximetry in the diagnosis of OSAS, ranging from 31$98 \%$ and $41-100 \%$, respectively [16], and its use is not recommended by current clinical guidelines [14].

\section{ECG analysis}

Monitoring of heart rate, and in particular heart rate variability, by overnight ECG has long been recognised as a potential diagnostic strategy in patients with suspected sleepdisordered breathing [17]. Other modalities utilising overnight ECG include analysis of dynamic changes in ECG morphology accompanying obstructive events, the accuracy of which may be significantly enhanced by the application of sophisticated diagnostic algorithms [18].

Another potentially promising strategy is the combination of ECG analysis with oximetry [19], but there is a general lack of highquality, empirical evidence on this subject, and the use of ECG analysis, with or without concomitant oximetry, is not currently endorsed by international guidelines [14].

\section{Novel diagnostic modalities}

Advances in technology have facilitated the development of a number of novel diagnostic strategies in the investigation of suspected OSAS. One of the best studied of these is the use of peripheral arterial tonometry, whereby peripheral vasoconstriction, caused by increased sympathetic tone, is recognised by a sensor applied to the subject's finger [20]. This approach has been validated in a number of studies, whether used in isolation, or in combination with oximetry and/or actigraphy [21]. Furthermore, this approach has the potential to allow estimation of future cardiovascular risk via the identification of endothelial dysfunction [22].

A more conceptually straightforward singlechannel device measures respiration via a nasal cannula that senses airflow, and hence generates an estimate of the degree of sleep disordered breathing. While clearly not as suitable for individuals with significant nasal obstruction or those who breathe largely though their mouth, prospective validation studies of this technology appear promising [23].

Other novel techniques include estimation of respiratory effort by measuring jaw movements or the use of oesophageal manometry to identify the intrathoracic pressure swings that characterise obstructive events [12]. Further evidence is needed to support use of the former, while the use of the latter approach is limited by its invasive nature [12].

All of the diagnostic modalities discussed above require the device used to be fitted to the subject, either by staff from the sleep laboratory or by the subject themself. A potentially attractive alternative approach invoIves the use of a noncontact, bedside device, which uses the reflection of radio waves to estimate sleep, respiratory effort and body movement via a sophisticated signal analysis algorithm [24]. While further prospective validation studies are pending, the available data suggest this device may prove to be a useful addition to the current diagnostic armamentarium [25].

\section{The cost-effectiveness of ambulatory diagnosis}

The entire rationale behind the use of ambulatory diagnostic strategies in OSAS is that they will lead to more efficient resource utilisation by sleep services, thereby facilitating the investigation and diagnosis of a greater number of individuals with the disorder. Unfortunately, there remains a significant lack of high-quality data assessing the economic impact of ambulatory diagnosis, with recent expert reviews advising the incorporation of rigorous economic analyses into future clinical trials in the area [6]. Cost-effectiveness is intimately related to two other vital, but incompletely answered, questions; first, which patients should undergo ambulatory testing, and second, does ambulatory testing lead to equivalent patient-centred outcomes to inpatient PSG?

\section{Patient selection for ambulatory diagnosis}

Although many of the technologies discussed above would appear to facilitate screening of large, unselected populations for undiagnosed sleep-disordered breathing, the need to perform further in-patient testing on any subjects 
with negative or inconclusive home studies would completely overwhelm existing sleep services [6]. Rather, the target population for ambulatory testing remains confined to subjects with a high pre-test suspicion of OSAS on clinical grounds [14]. However, even within this relatively restricted group, further examination of the utility of domiciliary testing remains necessary. In particular, there is a paucity of studies involving elderly patients, ethnic minorities, and patients with comorbid cardiac, pulmonary and neuromuscular disease [26]. A proposed algorithm for the use of ambulatory technologies is presented in figure 1.

\section{Outcomes following ambulatory diagnosis}

Prior studies have demonstrated the ability of nontraditional diagnostic strategies to achieve equivalent outcomes to in-patient PSG in the investigation of subjects referred with suspected OSAS [27]. Many recent validation studies show close correlations of PSC measurement of sleep-disordered breathing severity with proposed ambulatory methods. However, there is a general lack of studies addressing the effectiveness of ambulatory devices with regard to outcomes, and data exploring the performance characteristics of these devices in the appropriate clinical setting is also required before their widespread use can be recommended [26].

\section{Conclusion}

Waiting times for the diagnosis and treatment of OSAS in sleep laboratories are unacceptably long throughout the developed world. This situation is likely to deteriorate as awareness of the disorder increases among the public and the wider medical community.

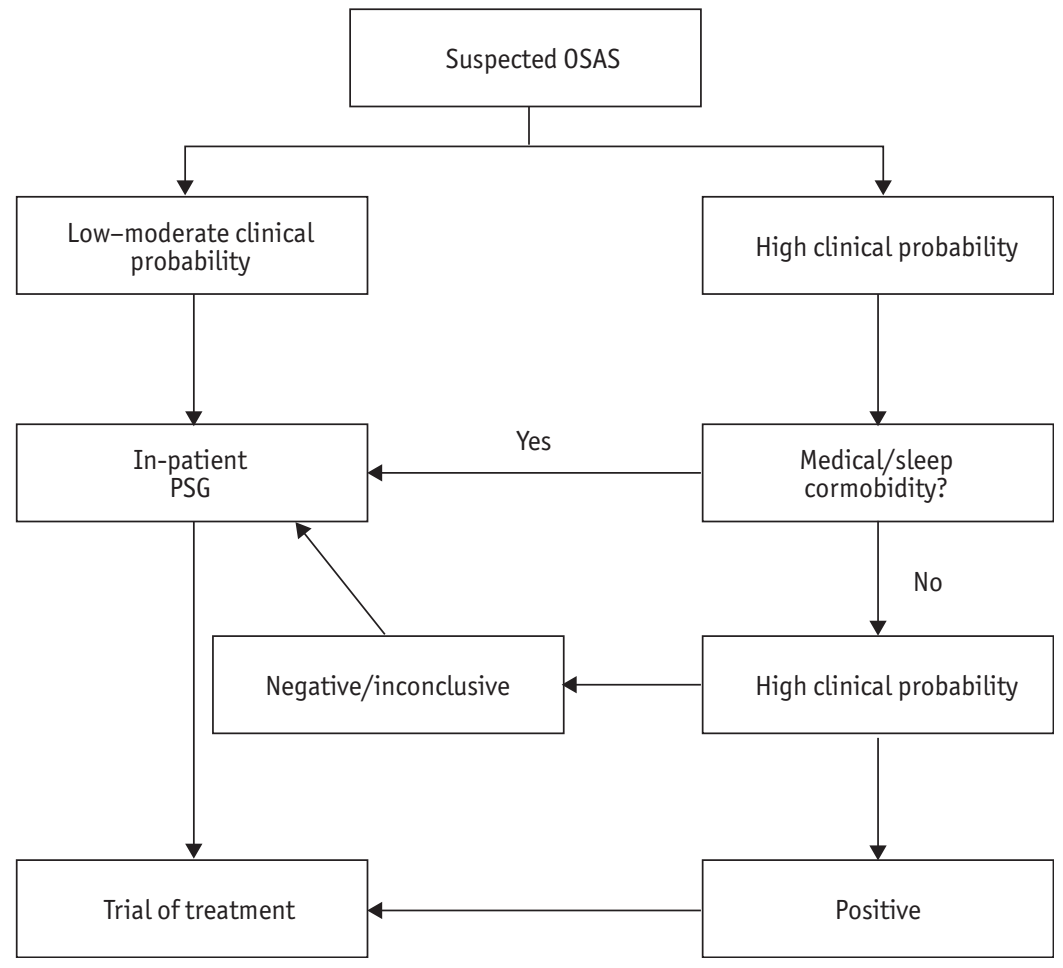

Figure 1

Proposed diagnostic algorithm for the ambulatory diagnosis of sleep-disordered breathing. OSAS: obstructive sleep apnoea syndrome; PSG: polysomnography.

Therefore, the identification and validation of new diagnostic modalities represents a research priority. While technological advances have permitted the development of potentially exciting new diagnostic tools, the available evidence base has lagged far behind. The current literature and expert guidelines suggest that the ambulatory investigation of suspected OSAS is feasible in selected patient groups, but further studies evaluating patient outcomes following ambulatory diagnosis are warranted. Furthermore, a thorough exploration of the economic impact of these devices on sleep services is urgently needed.
1. Young T, Palta M, Dempsey J, et al. The occurrence of sleep-disordered breathing among middle-aged adults. N Engl J Med 1993; 328: 1230-1235.

2. Doherty LS, Kiely JL, Swan V, et al. Long-term effects of nasal continuous positive airway pressure therapy on cardiovascular outcomes in sleep apnea syndrome. Chest 2005; 127: 2076-2084

3. Marin JM, Carrizo SJ, Vicente E, et al. Long-term cardiovascular outcomes in men with obstructive sleep apnoea-hypopnoea with or without treatment with continuous positive airway pressure: an observational study. Lancet 2005; 365: 1046-1053.

4. Punjabi NM, Shahar E, Redline S, et al. Sleepdisordered breathing, glucose intolerance, and insulin resistance: the Sleep Heart Health Study. Am J Epidemiol 2004; 160: 521-530.

5. Flemons WW, Douglas NJ, Kuna ST, et al. Access to diagnosis and treatment of patients with suspected sleep apnea. Am J Respir Crit Care Med 2004; 169: 668-672. 
6. Kuna ST, Badr MS, Kimoff RJ, et al. An official ATS/ AASM/ACCP/ERS workshop report: research priorities in ambulatory management of adults with obstructive sleep apnea. Proc Am Thorac Soc 2011; 8: $1-16$.

7. Young T. Epidemiology of obstructive sleep apnea: a population health perspective. Am J Respir Crit Care Med 2002; 165: 1217-1239.

8. Young T, Evans L, Finn L, et al. Estimation of the clinically diagnosed proportion of sleep apnea syndrome in middle-aged men and women. Sleep 1997; 20: 705-706.

9. Standards of Practice Committee of the American Sleep Disorders Association. Practice parameters for the use of portable recording in the assessment of obstructive sleep apnea. Sleep 1994; 17: 372-377.

10. Iber C, Redline S, Kaplan Gilpin AM, et al. Polysomnography performed in the unattended home versus the attended laboratory setting - Sleep Heart Health Study methodology. Sleep 2004; 27: $536-540$.

11. Portier F, Portmann A, Czernichow P, et al. Evaluation of home versus laboratory polysomnography in the diagnosis of sleep apnea syndrome. Am J Respir Crit Care Med 2000; 162: 814-818.

12. McNicholas W. Diagnosis of obstructive sleep apnea in adults. Proc Am Thorac Soc 2008; 5: 154-160.

13. Ross SD, Sheinhait IA, Harrison KJ, Kvasz M, Connelly JE, Shea SA, et al. Systematic review and meta-analysis of the literature regarding the diagnosis of sleep apnea. Sleep 2000; 23: 519-532.

14. Collop NA, Anderson WM, Boehlecke B, et al. Clinical guidelines for the use of unattended portable monitors in the diagnosis of obstructive sleep apnea in adult patients. Portable Monitoring Task Force of the American Academy of Sleep Medicine. J Clin Sleep Med 2007; 3: 737-747.

15. Whitelaw WA, Brant RF, Flemons WW. Clinical usefulness of home oximetry compared with polysomnography for assessment of sleep apnea. Am J Respir Crit Care Med 2005; 171: 188-193.

16. Netzer N, Eliasson AH, Netzer C, et al. Overnight pulse oximetry for sleep-disordered breathing in adults: a review. Chest 2001; 120: 625-633.
17. Guilleminault C, Connolly S, Winkle R, et al. Cyclical variation of the heart rate in sleep apnoea syndrome. Mechanisms, and usefulness of $24 \mathrm{~h}$ electrocardiography as a screening technique. Lancet 1984; 1 : 126-131.

18. Heneghan C, de Chazal P, Ryan S, et al. Electrocardiogram recording as a screening tool for sleep disordered breathing. J Clin Sleep Med 2008; 4: 223-228.

19. Heneghan C, Chua C-P, Garvey JF, et al. A portable automated assessment tool for sleep apnea using a combined Holter-oximeter. Sleep 2008; 31: 1432-1439.

20. Bar A, Pillar G, Dvir I, et al. Evaluation of a portable device based on peripheral arterial tone for unattended home sleep studies. Chest 2003; 123: 695-703.

21. Zou D, Grote L, Peker Y, et al. Validation a portable monitoring device for sleep apnea diagnosis in a population based cohort using synchronized home polysomnography. Sleep 2006; 29: 367-374.

22. Rubinshtein R, Kuvin JT, Soffler M, et al. Assessment of endothelial function by non-invasive peripheral arterial tonometry predicts late cardiovascular adverse events. Eur Heart J 2010; 31: 1142-1148.

23. Erman MK, Stewart D, Einhorn D, et al. Validation of the ApneaLink for the screening of sleep apnea: a novel and simple single-channel recording device. J Clin Sleep Med 2007; 3: 387-392.

24. De Chazal P, Fox N, O'Hare E, et al. Sleep/wake measurement using a non-contact biomotion sensor. J Sleep Res 2011; 20: 356-366.

25. Zaffaroni A, de Chazal P, Heneghan C, et al. SleepMinder: an innovative contact-free device for the estimation of the apnoea-hypopnoea index. Conf Proc IEEE Eng Med Biol Soc 2009; 2009: 7091-7094.

26. McNicholas WT, Lévy P. Portable monitoring in sleep apnoea: the way forward? Eur Respir J 2011; 37: 749-751.

27. Mulgrew AT, Fox N, Ayas NT, et al. Diagnosis and initial management of obstructive sleep apnea without polysomnography: a randomized validation study. Ann Intern Med 2007; 146: 157-166. 